\title{
Preface: numerical and applied linear algebra
}

\author{
Rafael Bru • Juan Manuel Peña
}

Published online: 6 July 2011

(c) Springer Science+Business Media, LLC 2011

This special issue of Advances in Computational Mathematics is devoted to Numerical and Applied Linear Algebra, which is an important area of mathematics and a fundamental part of engineering and computational science problems. One of the main tools of this area is matrices, properties of which are studied and computations of them are performed, with different methods, in a variety of applications. The special issue contains 12 papers reflecting some specialized themes on numerical and applied linear algebra and at the same time gives an idea of the broadness of this area, which has grown spectacularly in the last decades.

The work entitled "Structured linear algebra problems in adaptive optics imaging", by Johnathan M. Bardsley, Sarah Knepper, and James Nagyy, discusses an adaptive optics image problem to remove the effects of the phase error. For solving the Kronecker product structured, rank-deficient least-squares problem on the Fried geometry, two types of regularization are considered: a truncated singular value decomposition type and a Tikhonov type.

The paper "On the numerical solution of large-scale sparse discrete-time Riccati equations", by Peter Benner and Heike Faßbender, deals with the numerical solution of discrete algebraic Riccati equations. Since the systems considered are large-scale, the solution process for the computation of a

Communicated by Rafael Bru and Juan Manuel Peña.

R. Bru

Department of Applied, Mathematics of the Politechnical University of Valencia, Valencia, Spain

J. M. Peña $(\bowtie)$

Department of Applied Mathematics, University of Zaragoza, Zaragoza, Spain

e-mail: jmpena@unizar.es 
Newton step needs to be of iterative type. The authors develop new versions of a low rank Smith and low rank ADI methods to accomplish this task.

The paper entitled "A preconditioning technique for a class of PDEconstrained optimization problems", by Michele Benzi, Eldad Haber and Lauren Taralli, studies the use of a preconditioner for the saddle point system that arises in each inexact Gauss-Newton iteration applied to a discretization of the PDE-constrained optimization problem. The preconditioner is built using information obtained in the discretization steps. Further, spectral properties of the preconditioned matrix are given.

The work "A note on computing geometric means", by Dario A. Bini and Bruno Iannazzo, introduces a new (iterative) definition of a geometric mean (which they call cheap mean) of a finite set of positive definite matrices. Then they prove, under some hypothesis, cubic local convergence of the computational algorithm for this mean. They show that this mean satisfies most of the Ando-Li-Mathias properties of a "geometric mean". They give a numerical counterexample to the effect that their mean does not, in general, satisfy the monotonicity property.

The work "Minimizing and Maximizing the Euclidean Norm of the Product of Two Polynomials", by F. Bünger, considers the problem of minimizing or maximizing the quotient between the norm of the product of two polynomials and the product of the norms of both polynomials. These results are obtained by transferring the optimization problem to that of determining extremal eigenvalues and corresponding eigenvectors of autocorrelation Toeplitz matrices.

The paper "From approximating to interpolatory non-stationary subdivision schemes with the same generation properties", by Costanza Conti, Luca Gemignani and Lucia Romani, presents a general strategy to deduce a family of interpolatory subdivision schemes from a non-interpolatory subdivision scheme, in the non-stationary case. They also discuss some computational examples by showing that the proposed approach can yield novel smooth nonstationary interpolatory subdivision schemes possessing interesting reproduction properties.

In the work "Greville's method for preconditioning least squares problems", by Xiaoke Cui, Ken Hayami and Jun-Feng Yin, preconditioning full or rank deficient matrices of least squares problems is considered. The Greville's method is used to construct a sparse approximation to the Moore-Penrose inverse of $\mathrm{A}$. The equivalence between the least square preconditioned problem and the original problem is given and the breakdown is studied when the preconditioner is applied to GMRES. 
The paper "A simple generalization of Geršgorin's theorem", by Ljiljana Cvetković, Vladimir Kostić, Rafael Bru and Francisco Pedroche considers two classes of nonsingular matrices introduced by Ostrowski that are known as $\alpha 1$ matrices and $\alpha 2$-matrices, respectively. Both classes of matrices are defined in terms of a parameter $\alpha$ in $[0,1]$. This paper provides new characterizations of these classes of matrices that are independent of the value of the parameter $\alpha$. In addition to its intrinsic interest, this independence of the parameter $\alpha$ allows the authors to provide eigenvalue localization results of Geršgorin's type.

The work "QR methods and error analysis for computing Lyapunov and Sacker-Sell spectral intervals for linear differential-algebraic equation", by $\mathrm{Vu}$ Hoang Linh, Volker Mehrmann and Erik S. Van Vleck, deals with the study of numerical methods based on QR factorization for computing Lyapunov or Sacker-Sell spectral intervals for linear differential algebraic equations given in strangeness-free form. Further, a perturbation and error analysis of the spectral intervals obtained by these new QR methods are given.

The work "Finite-element based sparse approximate inverses for blockfactorized preconditioners", by Maya Neytcheva, Erik Bängtsson and Elisabeth Linnér, is devoted to the construction of algebraic multilevel preconditioners based on sparse approximate inverses. It analyses a method to construct numerically efficient and computationally cheap sparse approximations of some of the matrix blocks arising in the block-factorized preconditioners for matrices with a two-by-two block structure.

The paper "Diagonal dominance, Schur complements and some classes of H-matrices and P-matrices", by Juan Manuel Peña, introduces a new subclass of invertible $\mathrm{H}$-matrices (matrices that are strictly diagonally dominant by rows after scaling their columns). Only strict diagonally dominance in a row is imposed to define this new subclass. The Schur complement of this subclass is closed under suitable pivoting strategies. With this result a new subclass of matrices with all their principal minors positive (P-matrices) is presented.

The paper "Top-level acceleration of adaptative algebraic multilevel methods for steady-state solution to Markov chains", by H. De Sterck, K. Miller, T. Manteuffel and G. Sanders, discusses two acceleration techniques of multiplicative multilevel methods designed in the algebraic multigrid (AMG) framework when computing the steady-state distribution vector of a nonsymmetric irreducible Markov chain. The first acceleration technique minimizes an unconstrained functional and the second one uses a constrained minimization problem. 


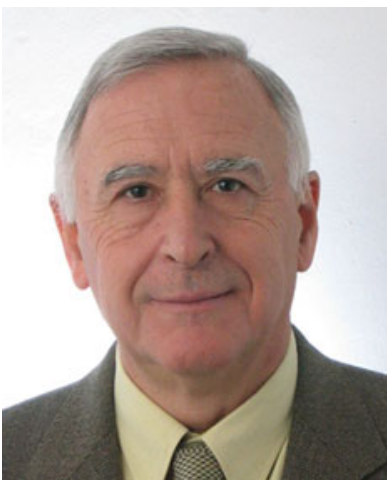

Rafael Bru is a Full Professor at the Department of Applied Mathematics of the Politechnical University of Valencia (Spain). He has been editor of special issues of the journals Linear Algebra and its Applications and Lecture Notes in Control and Information Sciences, and he is member of the Editorial Boards of the journals Advances Engineering Software and Proyecciones. Member of Organizing Committee of some Conferences on Linear Algebra and a Summer-School on Parallel Numerical Algorithms in Spain. Now he is the organizer of the 2012 SIAG/LA Conference on Applied Linear Algebra to be held in Valencia. His research interest are in Nonnegative Matrices and Numerical Linear Algebra mainly in Iterative Methods.

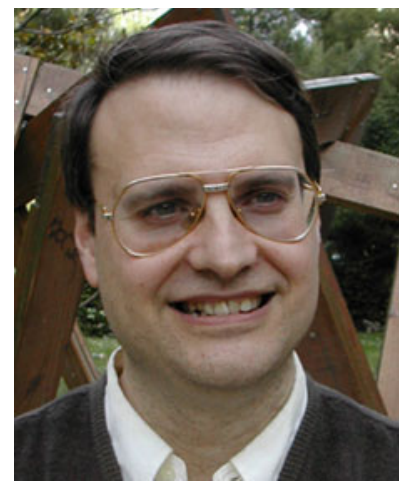

Juan Manuel Peña is a Full Professor at the Department of Applied Mathematics of the University of Zaragoza (Spain). He is author and co-author of more than 200 research papers. He has been editor of special issues of the journals Linear Algebra and its Applications, Advances in Computational Mathematics and Journal of Approximation Theory and he is member of the Editorial Boards of the journals Advances in Computational Mathematics and Journal of Applied Mathematics. His current research interests are Numerical Linear Algebra, Total Positivity, Approximation Theory and Computer-Aided Geometric Design. 